\title{
The Prevalence of Tuberculosis in Cattle and Buffalo in the Koshi Hills of Nepal
}

\author{
A. M. Morel
}

\begin{abstract}
The prevalence of tuberculosis in cattle and buffalo was estimated in Pakhribas panchayat using the Single Intradermal Comparative Tuberculin Test.

One percent of cattle and two percent of buffaloes were positive to the test with two percent of the buffaloes as inconclusive reactors.

The importance of tuberculosis as a zoonosis in this area is discussed.
\end{abstract}

\section{Introduction}

Tuberculosis is a major public health problem in the human population of Nepal. The prevalence of the disease in humans in this country is estimated to be 4-5 percent (smear positive), but the overall number of $X$-ray suspected cases may exceed 20 percent. It is believed that 60,000 people in Nepal may be sulfering from tuberculosis with an annuat mortality of 15,000 (Tuberculosis Control Project. 1985).

Perera and Crofton (1978)estimated the prevalence of smear positive cases based on active and passive case finding to be 3.6 per 1000 country wide, and in Sankhuwa Sabha District. KoshiZone, a British Nepal Medical Trust (BNMT) survey identified 4.03 cases per 1000 as being smear positive (Cassels, Heineman, LeClerq, Gurung and Rahut, 1982).

A sputum survey conducted in Dhankuta in 1975 gave an estimated prevalence of 1.91 positive tuberculosis cases per 1000 population and when considering sputum smear positive, sputum culture positive and $X$-ray positive cases suggested infection rates of 6.84 per 1000 for Dhenkuta and 6,7 cases per 1000 for the whole country (Perera and Crofton, 1978). 
All samplès submitted to the Scottish Mycobacterium Reference Laboratory by BNMT for identification from the Eastern Region of Nepal revealed Mycobacterium tuberculosis (BNMT, Pers. Comm., 1985). Similarly, all samples submitted to Milibank, England, by the British Medical Hospital. Dharan, have been of M. tuberculosis (BMH, Pers. Comm., 1985). No cases of humans suffering from $M$. bovis have yet been recorded and the vast majority of human cases have been of the puimonary form.

Little work has been done, to date, on tuberculosis in the animal population, but Singh (1971) identified 5-6 percent of buffalo slaughtered in Kathmandu abattoir as being tuberculosis positive on direct smear and histology.

Tuberculin testing of cattle and butfalo has been confined to government livestock farms. In 1974 Joshi, Shukla and Pyakuryal reported that of 39 . buffalo tested using the Double Intradermai Tuberculin Test (DID) at Pokhara government farm.2 were positive and 7 inconclusive reactors. Of 6 cattle tested, 1 was positive and inconclusive to the test. From 1975-1978, 10 percent of the buffalo and catte herds at Khumaitar. Tarahara and Pokhara government farms were positive to the DID (Singh. 1978) and in 1981 a further survey at Pokhara government farm gave 4.5 percent of the buffalo herd as positive reactors (Singh, 19821.

The following survey was conducted in Pakhribas panchayat, Dhankuta District, in order to assess the prevalence of tuberculosis in cattle and buffalo in the field.

\section{Materials and Methods}

According to Pakhribas Agricultural Centre Livestock Census Survey data (1984). Pakhribas panchayat contains 461 farm-holdings with a total population of 3360 cattle and 73 , buffalo. of these, 95 cattle and 45 buffalo from 53 farms were randomly selected for tuberculin testing.

The sample size selected for this survey was sufficient to give the true prevalence of tuberculin positive cattle within 10 percent at the 99 percent confidence level, assuming an expected prevalence of 10 percent and the true prevalence in buffaloes within 10 pescent ot the 95 percent confidence level, again assuming an expected prevalence of 10 percent (Canon and Roe, 1982).

The British Ministry of Agriculture, Fisheries and Food approved Single Intradermal Comparative Tuberculin Test (SID comparative) technique and interpretation was used in this survey - the test being read after 72 hours.

Positive and inconclusive reactors were clinically examined for nonspecific infection that could affect the test results (i. e. skin tuberculosis and Johne's disease). 


\section{Results}

The tuberculin test results are shown in table 1.

Table 1. Single Intradermal Comparative Tuberculin Test results in cattle and buffalo.

\begin{tabular}{|c|c|c|c|c|c|}
\hline & Number tested & $"+"$ & 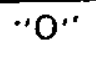 & $\because-\cdots$ & Prevalence (percent " + " ve) \\
\hline Cattle & 95 & 1 & 0 & 94 & 1 \\
\hline Buffalo & 45 & 1 & 1 & 43 & 2 \\
\hline $\begin{array}{l}"+"= \\
"-\ldots "= \\
" O "=\end{array}$ & $\begin{array}{l}\text { tuberculin posi } \\
\text { tuberculin nega } \\
\text { inconclusive }\end{array}$ & & & & \\
\hline
\end{tabular}

The positive and inconclusive reactors were alt from different farms.

The above results indicate a tuberculosis prevalence of 1 percent in cattle and 2 percent in buffalo (with 2 percent inconclusive reactors) as determined by the SID comparative test. None of the reactois showed clinical signs of skin tuberculosis or Johne's

disease.

\section{Conclusions}

This survey shows that the prevalence of tuberculosis in cattle and buffalo is at a low level in this area, and even if the inconclusive reactor is considered posittve, this still only gives a prevalence in buffalo of 4 percent-a considerably lower field prevalence in this area than the government rarm prevalence rates reported by Singh (1978;5982) and Joshi et al (1974).

Despite false positive and false negative reactors (Blood, Radostits and Henderson, 1983), the tuberculin test is a highly sensitive method of detecting tuberculous cattle-in one survey of 24,784 reactors, 98.39 percent showed macroscopic lesions on post-mortem investigation (Melvin, 1908).

Buffalo show a higher percentage of non-specific reactions (false positives) to the tuberculin test than cattle (Bratanovic and Ljesevic, 1974) with more pronounced and severe reactions and more extensive oedema (Lall, 1946: Awad and Mahmoud, 1957); thus the prevalence determined in this survey may be an over-estimato.

Although the prevalence in this area appears to be low, it might still be considered significant as a zoonosis were it not be for the fact that it is locai custoiti to boil all milk before consumption (milk being the major mode of transmission to man and inhalation only of secondary importance). The relative insignificance of cattle and buffalo tuberculosis as a zoonosis is born out by laboratory findings of $1 A$. Iuberculosis in humans, with no reports of $M$. bovis being isolated. The possibility of $M$. twbarculosis itself constituting an important zoonosis from cattle and buffalo to man is remote as these ungulates are relatively resjstant to infection with this organism (Blood, Radostits and Henderson, 1983; Robertson, 1976).

\section{Acknowledgements}

I would like to thank the Pakhribas panchayat field assistant for his help during this survey. 


\section{References}

Awad, F.I and Mahmoud, A.H. (1957) The Single Intradermal Comparative Tuberculin Test in the Egyptian Buffalo. Vet. Rec. 69: 133

Elood, D.C., Radositis, O.M. and Henderson, J.A. (1983) Veterinary Medicine, 631 \& 636 Balliere Tindall.

Bratanovic, U. and Liesevic. Z. (1974) cited by Lall, J.M. (1978)

Connon, R.M. and Roe, A.T. (1982) Livestock Disease Surveys: A field manual for Veterinarians. Austratian Government Publishing Service, Canberra.

Cossels, A., Heineman E. LeClenq. S., Curung, P.K. and nö́n̈t, C.B. (1982) Tuberculosis case-finding in Eastern :-ajai. Tubercle 63: 175-185

Ferers, H.W. and Crofton, J. (1978) Tuberculosis in Nepal. Assignment Report November 1977April 1978. WHO Project SEA/TB/148.

Lall. H.K. (1946) Tuberculin testing in buffaloes with a description of some differences in the macroscopic lesions of the cow and the buffalo. Indian J. Vet. Sci. 16: 24-26

LaII, J.M. (1978) Diagnosis of Tuberculosis in Animals. Indian Council of Agricultural Research, New Delhi.

Livestock Census Survey (1984) Pakhribas Agricultural Centre, Unpublished data.

Melvin A D. (1908) ciled by Lall, J M. (1978)

Ministry of Agriculture, Fisheries and Feed: Single Intradermal Comparative Tuberculin Test Technique and interpretation L.V.I. guidance notes.

Robertson A. Edit. (1976/ Handbook on Animal Diseases in the Tropics, 159. British Veterinary Association.

Singh, N.B. (1971) cited by Perera, H.W. annd Crofton, J. (1978).

Singh, N.B. (1978) Disease Investigation and Parasite Central Project (First Technical Report). HMG Nepal,

Singh. N.B. (1982) Annual Report of Animal Diseases and Parasite Control Division. HMG Nepal.

Joshi, D.D., Shukla, A.R. and Pyukural, S. (1974) Studies on Diseases of Domestic Animals and Birds in Nepal 111. Preliminary Observations on Tuberculosis, Para-tuberculosis and Brucellosis at the Livestock Farm, Pokhara. Bull. Vet. Sci and A. H. 3: 1-9.

Tuberculosis Contral Project (7985) Project Profosals for the Development of a Tuberculosis Control Programme in Nepal. HMG Nepal. 